VOL. $69(2004) \quad[137-140]$

\title{
ON RINGS WHOSE MODULES \\ OF FINITE LENGTH ARE ALL CYCLIC
}

\author{
YASUYUKI HIRANO
}

We give some characterisations of rings $R$ whose modules with composition series are all cyclic. In particular, we prove that all left $R$-modules of finite length are cyclic if and only if $R$ has no nonzero Artinian factor rings.

\section{INTRODUCTION}

In $D$-module theory, it is useful that every holonomic module over a Weyl algebra is cyclic and every holonomic module over the sheaf $D_{X}$ of holomorphic differential operators on a complex analytic manifold $X$ is locally cyclic (see [3, Corollary 2.6, Chapter 10] and [2, Proposition 3.1.5]). This follows from the fact that, if $R$ is a simple left Noetherian, non-Artinian ring and $M$ is a finitely generated Artinian left $R$-module, then $M$ is a cyclic module. (see [3, Theorem 2.5, p. 90] and [2, Proposition 1.1.35].) In this paper, we consider when the class of cyclic modules contains all modules of finite length. Let $R$ be a ring. We shall prove that every left $R$-module of finite length is cyclic if and only if $R$ has no simple left and right Artinian factor rings. Consequently we know that every left $R$-module of finite length is cyclic if and only if every right $R$-module of finite length is cyclic. We call a ring $R$ a finite length is cyclic-ring if $R$ satisfies these equivalent conditions. We shall give some charactrisations of a finite ring is cyclic-ring and using those, we shall prove that if $R$ is a finite ring is cyclic-ring, then every finite normalising extension of $R$ is also a finite ring is cyclic-ring.

\section{Modules of Finite LENGTH}

We begin with the following general considerations.

Let $M$ be a left $R$-module. For any subset $X$ of $M, \operatorname{Ann}_{R}(X)$ denotes the annihilator of $X$ in $R$. For each $m \in M$ and each submodule $N$ of $M$, we set $(N: m)=\{a \in R \mid$ $a m \in N\}$. For any positive integer $n, M^{(n)}$ denotes the direct sum of $n$ copies of $M$.

LEMMA 2.1. Let $R$ be a ring and let $M$ be a left $R$-module with composition series $M=M_{0} \supset M_{1} \supset \cdots \supset M_{n}=0$. If, for each $m \in M$, and each $i=1, \ldots, n-1$, $\left(M_{i+1}: m\right) \not \subset \operatorname{Ann}_{R}\left(M_{i} / M_{i+1}\right)$, then $M$ is cyclic.

Received 7th August, 2003

I would like to express my gratitude to the referee for his valuable comments.

Copyright Clearance Centre, Inc. Serial-fee code: 0004-9727/04 \$A2.00+0.00. 
Proof: Take $x \in M_{0} \backslash M_{1}$ and $y \in M_{1} \backslash M_{2}$. Then $R x+R y+M_{2}=M$. Since $\left(M_{2}: x\right) \not \subset \operatorname{Ann}_{R}\left(M_{1} / M_{2}\right)$ and since $R y+M_{2}=M_{1}$, there exists $a \in R$ such that $\left(M_{2}: x\right) a y+M_{2}=M_{1}$. Hence $R(x+a y)+M_{2}=M$. Continuing this process, we obtain $z \in M$ such that $R z=R z+M_{n}=M$.

PROPOSITION 2.2. Let $R$ be a ring and let $M$ be a left $R$-module of finite length. Suppose that, for each composition factor $N$ of $M$, the primitive factor ring $R / \operatorname{Ann}_{R}(N)$ is not left Artinian. Then $M$ is cyclic.

Proof: Consider a composition series $M=M_{0} \supset M_{1} \supset \cdots \supset M_{n}=0$. We shall prove that the condition in Lemma 2.1 is satisfied. Suppose, to the contrary, that $\left(M_{i+1}: m\right) \subset \operatorname{Ann}_{R}\left(M_{i} / M_{i+1}\right)$ for some $m \in M$ and $i$. Let $\bar{m}$ denote the homomorphic image of $m$ in $M / M_{i+1}$. Then $R \bar{m} \cong R /\left(M_{i+1}: m\right)$. Then $R / \operatorname{Ann}_{R}\left(M_{i} / M_{i+1}\right)$ is a homomorphic image of $R \bar{m}$, and hence it is left Artinian, a contradiction.

COROLlary 2.3. Let $R$ be a ring and let $M$ be a simple left $R$-module. If the primitive factor ring $R / \operatorname{Ann}_{R}(M)$ is not left Artinian, then $M^{(n)}$ is cyclic for any positive integer $n$.

We give some characterisations of rings all of whose modules of finite length are cyclic, which generalise [3, Theorem 2.5].

THEOREM 2.4. Let $R$ be a ring. Then the following statements are equivalent:

(1) Any left $R$-module of finite length is cyclic.

(2) There is a positive integer $n$ such that any left $R$-module of finite length is generated by $n$ elements.

(3) Every finitely cogenerated left $R$-module has an essential cyclic submodule.

(4) For any simple left $R$-module $M$ and any positive integer $n, M^{(n)}$ is cyclic.

(5) $R$ has no left Artinian factor rings.

(6) $R$ has no simple left Artinian factor rings.

$\left(1^{\prime}\right) \div\left(6^{\prime}\right)$ The left-right symmetric versions of (1)-(6).

Proof: The equivalence of (5) and (6) and the implications $(1) \Rightarrow(4)$ and $(1) \Rightarrow$ (2) are clear.

(1) $\Rightarrow(3)$. Let $X$ be a finitely cogenerated left $R$-module. Then $X$ has a finitely generated essential socle $S$ and $S$ is cyclic by hypothesis.

$(2) \Rightarrow(4)$. Assume that any left $R$-module of finite length is generated by $n$ elements. Let $M$ be a simple left $R$-module and consider the direct sum $M^{(m n)}$ of $m n$ copies of $M$. By hypothesis, there exists $n$ elements $x_{1}, \ldots, x_{n} \in M^{(m n)}$ which generate $M^{(m n)}$. For any left $R$-module $X$, let $L(X)$ denote the composition length of $X$. Then we have $m n=L\left(M^{(m n)}\right) \leqslant L\left(R x_{1}\right)+\cdots+L\left(R x_{n}\right)$. Hence, $L\left(R x_{i}\right) \geqslant m$ for some $i \in\{1, \ldots, n\}$. Since $R x_{i}$ is a submodule of the completely reducible module $M^{(m n)}$, this implies that $R x_{i} \cong M^{(m+k)}$ for some $k \geqslant 0$. Then $M^{(m)}\left(\cong M^{(m+k)} / M^{(k)}\right)$ is also cyclic. 
(3) $\Rightarrow(4)$. Let $M$ be a simple left $R$-module. Then, by hypothesis, $M^{(n)}$ has an essential cyclic submodule for any positive integer $n$. Since $M^{(n)}$ is completely reducible, this implies that $M^{(n)}$ itself is cyclic.

(6) $\Leftrightarrow\left(6^{\prime}\right)$. It is well-known that a ring is a left Artinian simple ring if and only if it is a right Artinian simple ring.

$(4) \Rightarrow(6)$. Suppose that $R / I$ is a left Artinian ring for some ideal $I$. We may assume that $R / I$ is a simple ring. Then we can easily see that $R / I \oplus R / I$ is not a cyclic left $R$-module.

(5) $\Rightarrow(1)$. This follows from Proposition 2.2. This completes the proof.

A ring $R$ is called a finite ring is cyclic-ring if $R$ satisfies the equivalent conditions of Theorem 2.4 .

COROLlaRY 2.5. Let $S$ be a finite normalising extension of a ring $R$. If $R$ is a finite ring is cyclic-ring, then $S$ is a finite ring is cyclic-ring.

Proof: This follows from Theorem 2.4 and [4, Corollary 10.1.11].

We note that the corollary above also follows more directly from [4, Proposition. 10.1.9(iii)].

Corollary 2.6. Let $S$ be an extension of a ring $R$ such that ${ }_{R} S$ is finitely generated and $S=R \oplus I$ where $I$ is a two-sided ideal of $S$. If $S$ is a finite ring is cyclic-ring, then $R$ is a finite ring is cycli-ring.

Proof: Let $K$ be a proper ideal of $R$. Then $S K S$ is an ideal of $S$ such that $S K S \cap R=K$. Hence $S / S K S$ is finitely generated as a left $R / K$-module. Since $S / S K S$ is not left Artinian, $R / K$ is also not left Artinian. Therefore this follows from Theorem 2.4 .

COROLlary 2.7. The class of finite ring is cyclic-rings is Morita stable.

Proof: This follows from the fact that the class of right Artinian rings are Morita stable (See $[\mathbf{1}$, Corollary 21.9]).

\section{REFERENCES}

[1] F.W. Anderson and K.R. Fuller, Rings and categories of modules, (second edition) (Springer-Verlag, New York, Heidelberg, Berlin).

[2] J. Björk, Analytic D-modules and Applications (Kluwer Academic Publishers, Dordrecht, Boston, London, 1993).

[3] S.C. Coutinho, A primer of algebraic D-modules, London Mathematical Society Student Texts 33 (Cambridge University Press, Cambridge, 1995).

[4] J.C. McConnell and J.C. Robson, Noncommutative Noetherian rings, Graduate Studies in Mathematics 30, (Revised edition) (American Mathematical Society, Providence, RI, 2001). 
Department of Mathematics

Okayama University

Okayama 700-8530

Japan

e.mail: yhirano@math.okayama-u.ac.jp 